\title{
A Robust system for Segmentation of primary Liver Tumor in CT images
}

\author{
Sonali Patil \\ PhD scholar, Shivaji University \\ faculty, University of Mumbai \\ K.J.Somaiya College of Engineering
}

\author{
V.R.Udupi, $\mathrm{PhD}$ \\ Visvesvaraya Technological University \\ Gogte Institute of Technology
}

\author{
Deepti Patole \\ University of Mumbai \\ $\mathrm{K} \mathrm{J}$ Somaiya College of Engineering
}

\begin{abstract}
The liver is a vital organ in human body, and Liver Tumor is considered to be a fatal disease. The tumors which can occur in Liver are cancerous or non-cancerous. For diagnosis of tumor, detection and demarcation of tumor is the initial step to be performed. After detection of the tumor, its type can be determined by using technique like biopsy, which is an invasive technique. To avoid such an invasive diagnosis technique, Non invasive techniques like diagnosis based on Medical Images using a CAD system can also be used. In such CAD systems, Detection and Segmentation of Tumor is performed automatically or semi-automatically. In this work, a system is developed to perform Segmentation of the Liver Tumor from abdominal CT image. This system segments the tumor in the two level operation. The first level of operation is segmentation of Liver from abdominal CT image and the second level is segmentation of Tumor from the result of first level. Segmentation of Liver is performed by using two methods namely Adaptive Thresholding with Morphological operations and Global Thresholding with morphological operations. Whereas segmentation of Tumor is performed by using three methods namely Adaptive Thresholding with Morphological operations, Fuzzy C Mean Clustering and Region Growing. This segmentation application generates and compares the outcomes of these implemented techniques. The system compares and selects the best of all the Tumor segmentation results and produces the final result. In this work, a robust system is proposed, by improving the accuracy of the segmentation for distinct quality and category of abdominal CT images, which contain liver tumors.
\end{abstract}

\section{General Terms:}

Benign, Malignant, Segmentation, Computer Tomography(CT) image, Computer Aided Diagnosis(CAD) System

\section{Keywords:}

Adaptive Thresholding, Mathematical Morphology, Global Thresholding, Region Growing, Fuzzy C Mean Clusteringifx

\section{INTRODUCTION}

The tumors, which occur in liver, can cause serious health issue. Such tumors can be of benign or malignant type. Non cancerous tumor masses which build inside liver region are called Benign or non-cancerous tumors. This kind of tumors are generally not life threatening. Where-as Cancerous tumor masses, which build inside liver region are known to be life threatening[1]. These Malignant or Cancerous Tumors need to be detected accurately for proper diagnosis and treatment. Correct diagnosis of such tumors at an early stage of disease helps to treat and save patient from fatal consequences. In this work detection and segmentation of such benign and malignant tumors from Abdominal CT images is implemented. The liver tumor segmentation has to be performed on abdominal CT image slice of the patient. This CT image is noisy in this work's context. This noise in the image can be due to poor image quality of the CT machine. Also the CT images from the same machine tend to vary in the intensity levels for different patients. In addition to these image quality related noises, there exist some inevitable complexities in the abdominal CT image. These other complexities are because of existence of ambiguous boundaries between all the adjacent organs in the abdominal CT image. The CT image contains soft organs like kidney, intestine, pancreas, spleen, abdominal wall etc. which may have intensity levels quite comparable to intensity levels of Liver. These all factors affect segmentation of tumor.

Hence, segmentation of liver tumor is performed by considering all these ambiguities, noise and inter patient variance [2] of intensity levels in the CT images. This work divides the segmentation of tumor into two parts viz. segmentation of Liver and segmentation of tumor from segmented liver. For better results, the CT images are preprocessed prior to actual segmentation process. The first part of segmentation i.e. Liver segmentation is implemented by using Adaptive thresholding, Global Thresholding and mathematical morphology. The result of the first stage of segmentation reduces the complexity level of the image by segmenting out the Liver, from rest of the abdominal adjacent organs. This first segmentation result is further processed by using Adaptive thresholding, Fuzzy $\mathrm{C}$ Mean Clustering and Region Growing. The execution of second stage of segmentation reveals the Tumor, segmented and extracted from the abdominal CT image.

The rest of this paper is organized as follows. Section 2 describes the related work, Section 3 discusses about the segmentation process. Section 4 presents the results along with the discussion and finally Section 5 concludes the work.

\section{RELATED WORK}

The detection of tumor for classification of benign and malignant types using data extracted from images has been an active research topic in recent years. For such research the images used are ultrasonic, CT, MRI etc. Various methods have been proposed and discussed for segmentation of these medical images. The segmentation of medical image needs to be performed in different manner for each type of image. Also the segmentation procedures differ largely based on the organ to be segmented.

In a research article, Mala et. al [3], have used adaptive thresholding technique for segmentation of liver region from CT image, and further used fuzzy $\mathrm{C}$ mean clustering algorithm for tumor segmentation. Author has suggested that such two stage 
segmentation has improved the result. Liver segmentation using graph cut based model [4], has been proposed by Raja S Alomari et. al. This approach combines Markov Random Fields [5] and Gradient Vector Field snakes [6] to provide segmentation of the liver tissue. Chen et. al [2], has proposed to use the prior knowledge of livers geometry and pixel level attributes unified to form the energy function via fuzzy similarity measure. The prior knowledge based approach with Markov Random Fields [5] and Gradient Vector Field snakes [6], provides segmentation of the liver tissue. In their method, the only input required from the user is a single pixel inside the liver. This results in human intervention in the segmentation process. Human intervention can create a limitation in full automation of the system. In order to fully automate the segmentation process some assumptions have to be made in terms of image content, which may introduce some error in the segmentation results. It is suggested in article [4] that, the error in such results may result in catastrophic mistakes in the whole diagnosis process. Another simple approach towards full automation of segmentation process is by making use of histogram [7]. The liver is largest and fairly homogeneous organ visible in abdominal CT image, which shows liver, Hence the histogram shows a peak, related to pixels of liver area. This peak of the histogram can be used for liver segmentation. But this method fails when in CT image, the visibility of liver is not the largest of all other organs, due to change in position of 2D CT slice or when there exists a huge tumor in liver region with different texture and different intensity levels than that of the Liver. The article [8] has presented an algorithm for the segmentation of liver metastases in CT scans. It is a hybrid method that combines adaptive thresholding based on a gray value analysis of the ROI with model-based morphological processing. A semi-automatic method based on 2D region growing with knowledge-based constraints [9] has been proposed by Wong et. all to segment lesions from constituent 2D slices obtained from 3D CT images. Minimal user involvement is required to define an approximate region of interest around the suspected legion area. Then, the seed point and feature vectors have been calculated. Voxels [10] are labeled using a region-growing approach. Prior knowledge-based constraints have been incorporated into the method to ensure that, the size and shape of the segmented region is within acceptable parameters. [9]. But, the abdominal $\mathrm{CT}$ images vary as the position of slice varies. Hence, the prior knowledge can prove to be deceiving. Therefore, it can be stated that there exists a practical constraint in setting knowledge based attributes, necessary for full automation of the system.

\section{SEGMENTATION OF LIVER TUMOR}

The proposed system is designed to have four steps. First step is preprocessing of image and second step is segmentation of image to extract liver region and further extracting tumor area from extracted liver region. The proposed system is as shown in figure 1.

The CT and MRI images of abdomen which show liver region, consist of imagery of other soft tissue organs like spleen, intestine, pancreas, and kidney. These all organs can have more or less similar gray levels in image. This fact makes liver segmentation complex. The variation in imaging machine's resolution and quality, leads to variation in gray levels of CT images. This can affect segmentation results. Hence for the purpose of image segmentation of grayscale CT abdominal image, it is necessary to preprocess the image.

\subsection{Preprocessing}

It has been observed that the $\mathrm{CT}$ images from different machines have different sizes. Images also contain some text written about the image and its other attributes. In addition to that, the ratio of

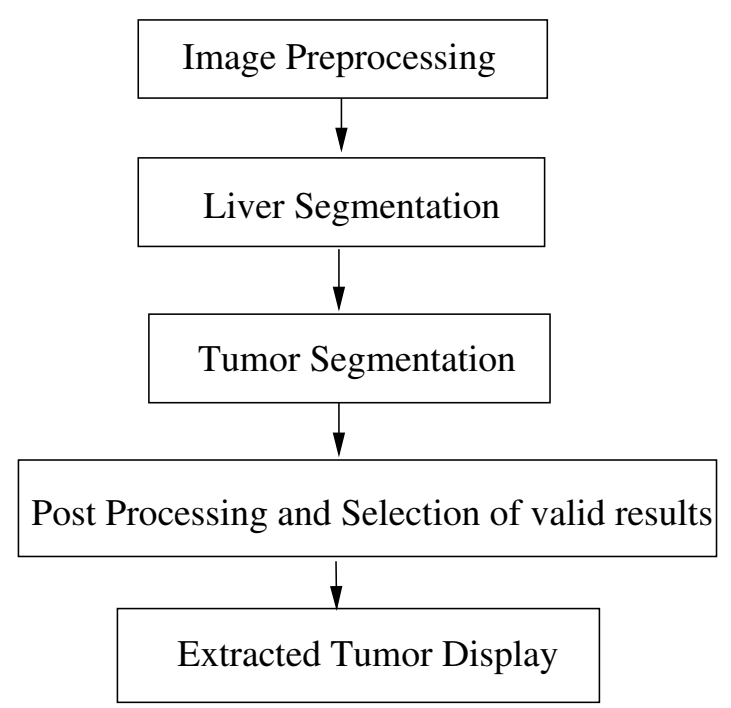

Fig. 1. System Flowchart

area of whole image to the area of region of interest in the total image, i. e. the actual body slice region, is not fixed for all the CT machines. This can include error in tumor size determination. In this work the preprocessing is performed, firstly by removal of any text present in the image and resizing the images to a common size so that tumor size can be determined. Secondly, if necessary, enhancing the contrast so that the organs can be distinctly segmented. These preprocessed images are further used as input for image segmentation.

\subsection{Segmentation}

\subsection{1 . Liver segmentation:}

The anatomical information of liver such as its size, location and structure helps in extraction of liver region from rest of organs. It has been found out from imagery representation of abdominal CT images which consist of Liver region that, the liver is the largest organ as far as imagery representation is concerned [3]. Its physical location is upper left corner of the image slice and due to its homogeneous structure it shows fairly homogeneous gray level over whole liver region. Liver is segmented, by making use of prior knowledge about size, location and gray level of Liver. For liver segmentation, methods such as global thresholding [11] and adaptive thresholding [8] along with morphology [8] are implemented.

3.2.1.1 . Liver segmentation by using Adaptive Thresholding and Morphology: This method of segmentation, separates foreground from background in an image with nonuniform gray-levels. The threshold for segmentation is calculated for smaller size windows across whole image. This thresholding results in a black and white image. This black and white image is then processed with morphological operations. And finally the largest distinct segment which lies in top-left region of the CT image is retained in the image, while removing all the extra segments present in the black and white image. This black and white image combined with original CT image results in segmented Liver region.

3.2.1.2 . Liver segmentation by using Global Thresholding and Morphology: In this method of segmentation a global threshold is selected depending upon overall intensity level of the CT image. This threshold is used for separating foreground pixels from background pixels. This separation results in black and white image. This black and white image is processed with morphology in similar way mentioned in section 3.2.1.1. Considering prior knowledge attributes of position and size of 
Liver in abdominal CT image, final Liver segmentation result is generated, as mentioned in section 3.2.1.1.

\subsection{2 . Tumor segmentation:}

Input to this stage of segmentation is the segmented Liver image. Tumor region in segmented liver has different gray level than that of liver. This allows tumor segmentation from input image which is segmented liver in this case. For tumor segmentation three techniques are used namely adaptive thresholding, fuzzy C mean clustering and region growing.

3.2.2.1 . Tumor segmentation by using Adaptive Thresholding and Morphology: The segmentation of Tumor, Adaptive thresholding is performed in similar way as mentioned in section 3.2.1.1. Morphological operations are performed to separate out the tumor region properly. The intensity level of the Tumor pixels can be more or less than that of Liver pixels. This fact causes tumor pixels to be considered as either background pixels or foreground pixels. If these pixels are foreground, tumor segmentation gives positive result, i.e. presence of tumor in the resultant image and absence of rest of Liver region. But, if the tumor pixels fall in background, The resultant image becomes negative i.e. absence of Tumor and presence of rest of Liver region in resultant image. The Tumor intensity can not be predicted to be lower or higher than that of Liver. In order to enhance the efficiency of the system, the complement of the resultant image is also considered as one of the Tumor segmentation results. Thus, either actual result or complement result can be considered as the valid Segmented Tumor Result

3.2.2.2 . Fuzzy C Mean Clustering: The fuzzy C mean clustering algorithm [12] [13] is implemented which outputs the binary image and threshold level of image. This binary image is multiplied with the segmented Liver image to extract Tumor from Liver region. The complement of the resultant image is also considered as separate Tumor segmentation result.

3.2.2.3 . Region Growing: A seed pixel is selected which lies in Tumor region of the CT image, this seed pixel intensity is compared with its neighboring pixels intensity and region is grown by using Region Growing Algorithm [14]

\subsection{3 . Post-processing}

The tumor segmentation stage results sometimes consist of segments of non-tumor regions of liver along with actual tumor region. These unwanted segments need to be removed from the image under process. In this work post-processing is implemented to remove all other non-tumor segments from the tumor segmentation stage result. These post-processing operations result into segmented Liver Tumor images.

\subsection{4 . Selection of valid result:}

Liver tumors are of different types and they vary in structure, gray level and size. Hence, the segmentation results tend to vary for the same CT image, when that image is processed with these implemented segmentation methodologies. This fact makes it necessary to choose the best result amongst all the Tumor segmentation stage results. For automatic selection of the best result , the area of tumor and existence of seed pixel in the result are considered as decisive factors. But these factors can cause error, if the tumor is too big. Hence it is suggested that, the final result should be validated by the radiologist. As an alternative method to this automatic selection of the final result, the implemented system has the facility of manual selection of the final result amongst all the post processed tumor results.

\subsection{Algorithm}

The system is designed with following algorithm. This algorithm presents systematic image processing methodology, to produce the final segmented Liver Tumor image as an outcome. step 1: Preprocessing of Image

step 2: Liver Segmentation using Global thresholding and Morphology

step 3: Liver Segmentation using Adaptive thresholding and Morphology

step 4: Selection of better result from 'Liver Segmentation using Global thresholding and Morphology' and 'Liver Segmentation using adaptive thresholding and Morphology'

step 5: Tumor Segmentation using adaptive thresholding

step 6: Tumor Segmentation using Fuzzy C mean Clustering

step 7: Tumor Segmentation using Region Growing

step 8: Complementing the adaptive thresholding and Fuzzy C mean Clustering results.

step 9: Post processing on the results of previous four steps.

step 10: Selection of better result from resultant images from five resultant images namely (a)adaptive Thresholding ,(b)Fuzzy C Mean Clustering, (c)Region Growing (d)complement of adaptive Thresholding and (e)complement of Fuzzy C Mean Clustering.

step 11: Display of results.

\section{RESULTS AND DISCUSSION}

The segmentation of CT image is performed by using five different techniques namely Adaptive Thresholding [8], Global Thresholding [11], Morphology [8], Fuzzy C mean Clustering and Region Growing [9].
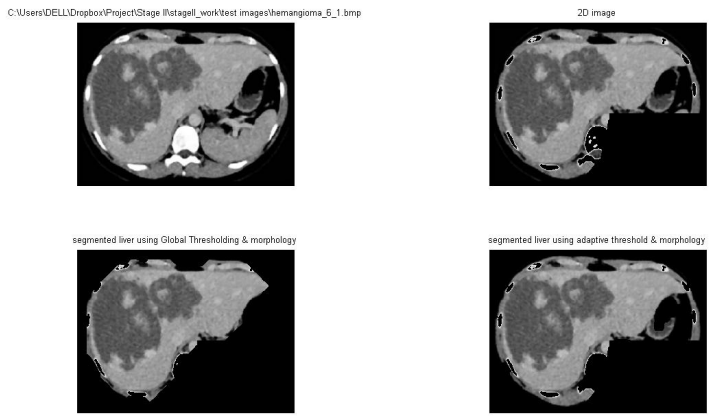

Fig. 2. a) Abdominal CT image,b) Preprocessed CT image,c) Liver Segmentation using Global thresholding and Morphology and d) Liver Segmentation using adaptive thresholding and Morphology
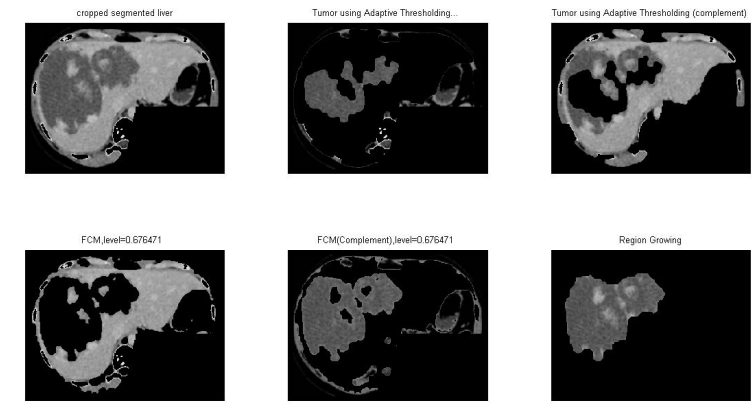

Fig. 3. a) Segmented Liver, b) Tumor Segmentation using Adaptive Thresholding and Morphology,c) Fuzzy C Mean Clustering and d) Region Growing 


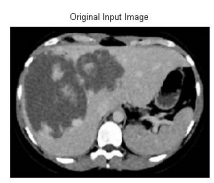

Results of tumor segmentation
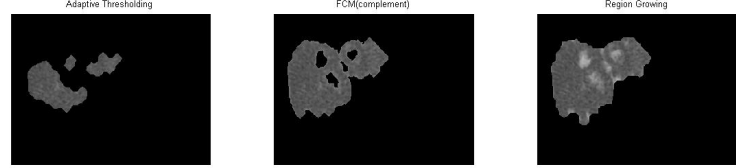

Fig. 4. Post Processed segmented Tumor showing images of a) Abdominal CT scan and Tumor Segmentation using b) Adaptive

Thresholding and Morphology, c) Fuzzy C Mean Clustering Complement and d) Region growing

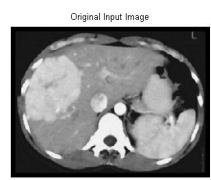

Results of tumor segmentation
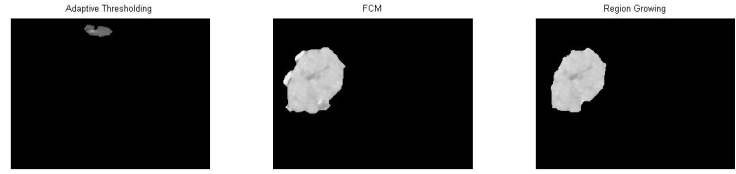

Fig. 5. Post Processed segmented Tumor showing images of a) Abdominal CT scan and Tumor Segmentation using b) Adaptive Thresholding and Morphology, c) Fuzzy C Mean Clustering and d) Region growing

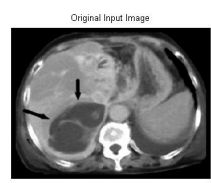

Results of tumor segmentation
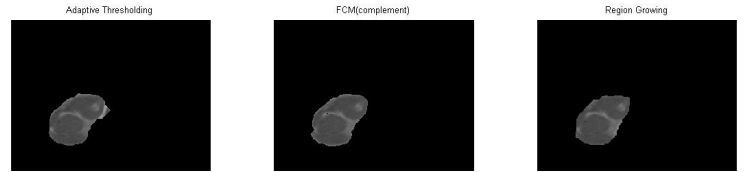

Fig. 6. Post Processed segmented Tumor showing images of a) Abdominal CT scan and Tumor Segmentation using b) Adaptive Thresholding and Morphology, c) Fuzzy C Mean Clustering Complement and d) Region growing

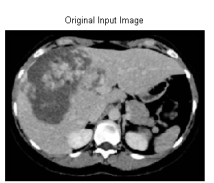

Results of tumor segmentation
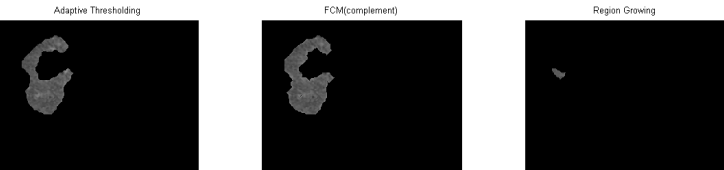

Fig. 7. Post Processed segmented Tumor showing images of a) Abdominal CT scan and Tumor Segmentation using b) Adaptive

Thresholding and Morphology, c) Fuzzy C Mean Clustering Complement and d) Region growing

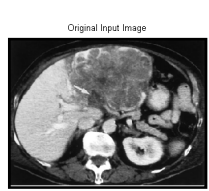

Results of tumor segmentation
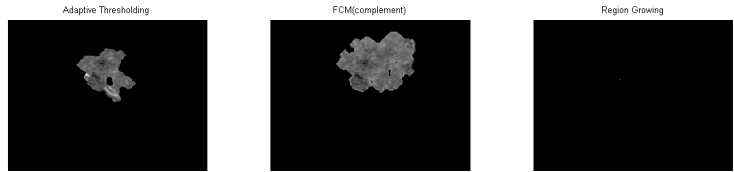

Fig. 8. Post Processed segmented Tumor showing images of a) Abdominal CT scan and Tumor Segmentation using b) Adaptive Thresholding and Morphology, c) Fuzzy C Mean Clustering Complement and d)Region growing

The first stage of segmentation is segmentation of the Liver region from rest of the CT image. Figure 2 shows the preprocessing and liver segmentation results. Preprocessing is performed by converting image to grayscale, removing any bone white regions present in image and applying a mask on the image which is designed by considering geometric attributes of the liver. Figure 3 shows output of third step of the system flowchart,as shown in figure 1 . Figure 4, shows the post processed result, i. e. final Tumor segmentation result. Figure 5, figure 6, figure 7 and figure 8 show the final results of segmentation by different techniques on four distinct abdominal $\mathrm{CT}$ images consisting of liver tumors.

Table 1. Comparison of Efficiency of different segmentation techniques

\begin{tabular}{|c||c|}
\hline Segmentation Technique & $\begin{array}{l}\text { Efficiency } \\
\text { segmentation } \\
\text { Liver Tumor }\end{array}$ \\
\hline Adaptive Thresholding & $37.69 \%$ \\
\hline ofaptive Thresholding (Complement) & $4.41 \%$ \\
\hline Fuzzy C Mean Clustering & $22.42 \%$ \\
\hline Fuzzy C Mean Clustering (Complement) & $37.22 \%$ \\
\hline Region Growing & $32.00 \%$ \\
\hline Proposed System & $92.3 \%$ \\
\hline
\end{tabular}

For carrying out the segmentation a dataset of 70 abdominal CT images consisting of liver tumors, has been built. From the results of the implementation, it has been observed that Fuzzy C 
mean Clustering works automatically and the results are equally good for lesser intensity tumors as well as higher intensity Tumors. Adaptive thresholding results are less erroneous if tumor intensity level is lesser than that of liver region but are erroneous if tumor intensity level is higher than that of liver. Region Growing Technique generates error free results if tumor is homogeneous, but does result in erroneous outcome if tumor itself has got intensity variations. Table 1 shows efficiency of different segmentation algorithms, dealt in this work for dataset of 70 abdominal CT images consisting of liver tumors. It has been observed that, as the tumor's structural properties vary, the segmentation results also tend to vary. It is clear from table 1 that, no single segmentation method is sufficient for segmenting the tumor in the Liver. The proposed system for segmentation of liver tumor from abdominal CT images consisting of liver tumor, results into much higher efficiency than any of the algorithms dealt in this work. This has provided the robustness of result generation irrespective of tumor type.

\section{CONCLUSION}

In this work, the segmentation of liver tumor from abdominal CT image is implemented. It has been observed that, the segmentation is affected by factors like inherent organ complexities, machine quality variations and inter-patient intensity variation. These inherent factors introduce error in segmentation. Improper segmentation leads to errors in classification of tumor. To avoid such unfortunate situation, the system has provided segmentation by making use of more than one method. This has given robustness to the system. The above factors affect the accuracy of segmentation by different algorithms in different proportion. The fact of of getting accurate segmentation result by atleast one of the implemented methods, has led to the development of the proposed system. The corresponding outputs of these algorithms present better opportunity for selection of error free or least erroneous result. In order to achieve least possible human intervention, segmentation methods such as Global thresholding using automatic threshold, Adaptive thresholding, Fuzzy C Mean Clustering and Mathematical Morphology are implemented. It has been observed from the work done that, complete elimination of human intervention causes false segmentation of tumor. Hence, to improve accuracy of the result a slight human intervention is included for seed pixel selection. This seed pixel is used for Region Growing Technique and for improving the accuracy of other implemented techniques. Hence this work presents a semi automatic system for the segmentation of Liver Tumor from CT scan images by making use of optimum human intervention for improving the accuracy of the results.

\section{REFERENCES}

[1] kanziuscancerresearch.org. Types of cancer being investigated: Liver cancer. http://www. kanziuscancerresearch.org/research/ types-of-cancer-being-investigated/ liver-cancer?gclid=CMy-m8anxbcCFY9V4god718AFw month $=$ August, year $=2013$,

[2] Yufei Chen, Weidong Zhao, Qidi Wu, Zhicheng Wang, and Jinyong Hu. Liver segmentation in ct images for intervention using a graph-cut based model. In Hiroyuki Yoshida, Georgios Sakas, and MariusGeorge Linguraru, editors, Abdominal Imaging. Computational and Clinicpd Applications, volume 7029 of Lecture Notes in Computer Science, pages 157-164. Springer Berlin Heidelberg, 2012.

[3] V.Sadasivam K.Mala and S.Alagappan. Neural network based texture analysis of liver tumor from computed tomography images. International Journal of Biological and Life Sciences 2:1, 2006.

[4] Vipin Chaudhary Raja S Alomari, Suryaprakash Kompalli. Segmentation of the liver from abdominal ct using markov random field model and gvf snakes. International Conference on Complex, Intelligent and Software Intensive Systems, IEEE, Octber 2008

[5] Z. Kato and J. Zerubia. Markov random fields in image segmentation. Foundations and Trends in Signal Processing, 5, 2012.

[6] Chenyang Xu and J.L. Prince. Snakes, shapes, and gradient vector flow. Image Processing, IEEE Transactions on, 7(3):359-369, 1998.

[7] Kyung-Sik Seo, Hyung-Bum Kim, Taesu Park, Pan-Koo Kim, and Jong-An Park. Automatic liver segmentation of contrast enhanced ct images based on histogram processing. In Lipo Wang, Ke Chen, and YewSoon Ong, editors, Advances in Natural Computation, volume 3610 of Lecture Notes in Computer Science, pages 1027-1030. Springer Berlin Heidelberg, 2005.

[8] J. Moltz, L. Bornemann, V. Dicken, and H. Peitgen. Segmentation of liver metastases in ct scans by adaptive thresholding and morphological processing. 072008.

[9] D. Wong, J. Liu, F. Yin, Q. Tian, W. Xiong, J. Zhou, Q. Yingyi, T. Han, S. Venkatesh, and S. Wang. A semi-automated method for liver tumor segmentation based on $2 \mathrm{~d}$ region growing with. 072008.

[10] T. Ben Said, O. Azaiz, F. Chaieb, S. M'Hiri, F. Ghorbel, and O. Azaiz. Segmentation of liver tumor using hmrf-em algorithm with bootstrap resampling. In I/V Communications and Mobile Network (ISVC), 2010 5th International Symposium on, pages 1-4, 2010.

[11] S. Wu and A. Amin. Automatic thresholding of gray-level using multistage approach. In Document Analysis and Recognition, 2003. Proceedings. Seventh International Conference on, pages 493-497 vol.1, 2003.

[12] Dzung L. Pham, Chenyang Xu, and Jerry L. Prince. Current methods in medical image segmentation1. Annual Review of Biomedical Engineering, 2(1):315-337, 2000. PMID: 11701515.

[13] Miin-Shen Yang, Yu-Jen Hu, Karen Chia-Ren Lin, and Charles Chia-Lee Lin. Segmentation techniques for tissue differentiation in $\{$ MRI $\}$ of ophthalmology using fuzzy clustering algorithms. Magnetic Resonance Imaging, 20(2): 173 - 179, 2002.

[14] Min Li, Hongyan Luo, Renbin He, Wenwu Zhu, Liwen Tan, and Yi Wu. Segmentation of white matter based on region growing and threshold theory. In Bioinformatics and Biomedical Engineering ( $i C B B E), 2010$ 4th International Conference on, pages 1-4, 2010. 